\title{
Communications
}

\section{Development of a Three-Channel, 24-h Ambulatory Esophageal Pressure Monitor}

CATHERINE J. PFISTER, MARK A. HARRISON, JOHN W. HAMILTON, WILLIS J. TOMPKINS, AND JOHN G. WEBSTER

\begin{abstract}
We have developed a three-channel ambulatory esophageal pressure monitor and tested it with a series of $24-h$ studies. The monitor is a battery-operated, microprocessor-based device that measures pressures from three transducers positioned in the esophagus, stores the data in its memory, and transfers the data to an IBM PC computer system at the end of the recording period. Programs on the PC then analyze the data and identify contractile events, categorizing them according to specific parameters. Other programs display the pressure waveforms on the $\mathrm{PC}$ and allow visual inspection of the entire recordings or, alternatively, of particular events of interest. The system detects contractile abnormalities in patients with intermittent, noncardiac chest pains. We tested the system on ten normal subjects and found a relatively high incidence of what are usually considered "abnormal" contractions.
\end{abstract}

\section{INTRODUCTION}

Many patients who report intermittent recurrence of severe angina-like chest pain test negative on standard cardiac evaluations such as exercise ECG and thallium scanning. In light of reports of an association between chest pain and esophageal motility disorders such as high-amplitude or tertiary contractions [1]-[6], the esophagus is suspected as being the source of pain in many such cases.

Conventional esophageal manometry, consisting of a brief stationary monitoring period, is unlikely to detect an episode of infrequently occurring pain. Pharmacologic provocation has been used, but has not added greatly to the sensitivity of esophageal motility [7]-[12]. An alternative approach would be to develop an ambulatory, long-term recording device where the esophageal pressure is monitored as the patient pursues normal activities in the home and elsewhere. Cassette tape recorder-based devices have already been developed for 24-h measurement of intraesophageal pressure [13], [14]. With such a device, the tape must be played back at the end of the recording period in order to analyze the data.

Digital recording, in contrast to analog recording, has the advantage of speed and ease of data transfer and evaluation without requiring an expensive piece of equipment, as does the Holter monitor. In addition, digital recording has the ability to do calculations and make decisions during the recording period, such as discarding unessential data points.

Computer analysis of esophageal pressure data is difficult, given the large variation in the appearance of a peristaltic wave and the number of "abnormal" contractile events that occur even in the normal human esophagus. Previous analysis by others of 24-h nonambulatory esophageal pressure data using a microcomputer compared fairly well to manual scoring, but no attempt was made to

Manuscript received February 10, 1988; revised September 26, 1988.

C. J. Pfister, W. J. Tompkins, and J. G. Webster are with the Department of Electrical and Computer Engineering, University of WisconsinMadison, Madison, WI 53706

M. A. Harrison and J. W. Hamilton are with the Department of Medicine, University of Wisconsin-Madison, Madison, WI 53706.

IEEE Log Number 8825624. differentiate between and classify the various contractile events [15].

We have developed a microprocessor-based system that allows 24-h ambulatory monitoring of three channels of esophageal pressure. The data are stored in random access memory (RAM) over the 24-h period and can subsequently be transferred in a few minutes to an IBM PC which processes and analyzes the data. We have tested a prototype wire-wrapped device on ten healthy, normal volunteers. In the future, we will use the monitor to evaluate patients with chest pain of noncardiac origin.

\section{Materials and Methods}

We interfaced a three-channel strain gage medical pressure catheter (Millar Instruments, Inc., Houston, TX) to our portable, bat tery-operated esophageal pressure monitor. The catheter is designed for use in standard stationary manometry testing, but can be easily connected to our system. The catheter contains three semiconductor microstrain-gage pressure transducers of sensitivity 5 $\mu \mathrm{V} / \mathrm{V}$ supply $/ \mathrm{mmHg}$. The transducers are spaced $5 \mathrm{~cm}$ apart and are housed in a $2.2-\mathrm{mm}$ diameter woven Dacron tube.

Fig. 1 shows the block diagram for the portable system. The voltages from the strain gage transducers are amplified by highgain, high-precision instrumentation amplifiers (INA101, BurrBrown, Tuscon, AZ) which have gains settable with a single external resistor. The 3-dB bandwidth is set from dc to approximately $2 \mathrm{~Hz}$. The amplifiers have very low temperature drift so that exposing the system to outdoor temperature extremes will not significantly affect the output. The amplifiers are zeroed by adjusting a potentiometer for each channel.

The amplified signals are then connected to an 8-bit analog-todigital converter (ADC) with a built-in 8-channel multiplexer (ADC0808, National Semiconductor Corporation, Santa Clara, CA). Sample-and-hold circuits are not needed due to the slowly varying pressure signal. The pressure signal on each channel is sampled at $5 \mathrm{sps}$, each conversion requiring $100 \mu$ s for completion. The system is based on an 8-bit CMOS microprocessor (Hitachi HD64180) which has several on-chip peripherals that facilitate the design of the system and reduce component count and power consumption. To extend battery life, the microprocessor goes into a SLEEP mode when it is not storing data or performing other tasks. It also shuts off the power to the strain gages and amplifiers between conversions. The device runs for $24 \mathrm{~h}$ on six alkaline AA batteries.

If all the data from the three channels were stored, the memory would be saturated in less than $3 \mathrm{~h}$. It is not possible to address enough memory to accomplish total storage of the 24-h pressure waveforms. Therefore, we save only those pressure data points above a predetermined threshold of $10 \mathrm{mmHg}$, along with $1 \mathrm{~s}$ of data prior to threshold crossing and $1 \mathrm{~s}$ after falling below threshold. This approach captures a complete peristaltic wave without wasting memory for baseline storage.

The transfer of the data to the IBM PC is initiated by a program written in the $\mathrm{C}$ language on the $\mathrm{PC}$ which sends a command via the serial port of the microprocessor in the monitor. The transfer of all 128 kbytes of memory is completed in 2-3 min at 9600 baud. The IBM then stores the data in a disk file. Other programs written in $C$ analyze the data and display the three channels on the monitor of the PC. Specific screens of interest are printed out on a graphics printer.

We simultaneously measured esophageal pressure using our portable system and a chart recorder and established that the portable system could faithfully reproduce the signal. Fig. 2 shows a sample of the signal obtained by the portable system. 


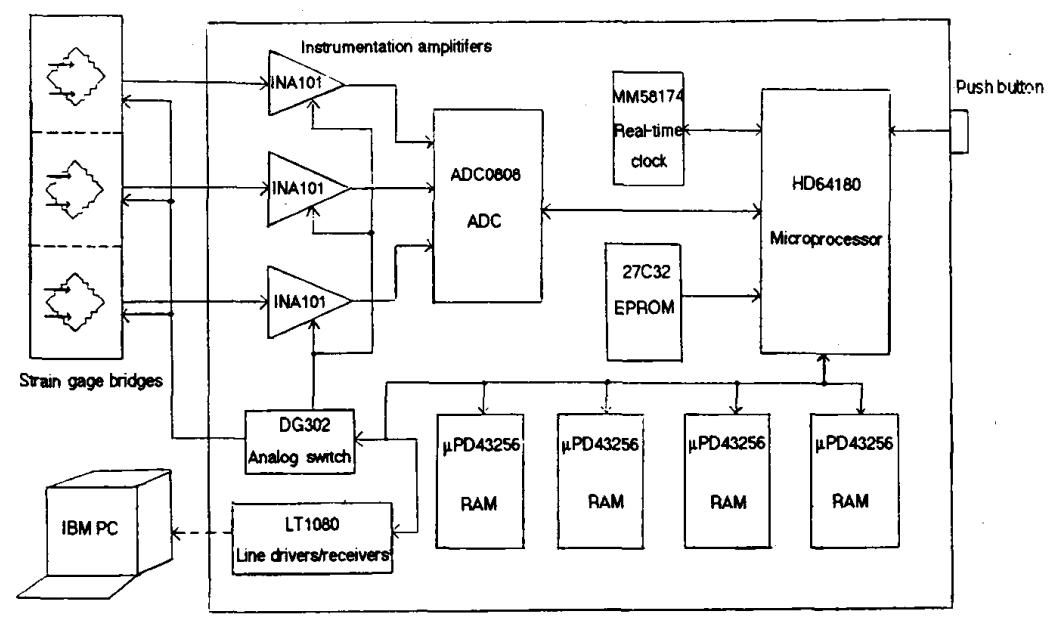

Fig. 1. The ambulatory esophageal pressure monitor acquires data for 24 $h$, then dumps it to an IBM PC.

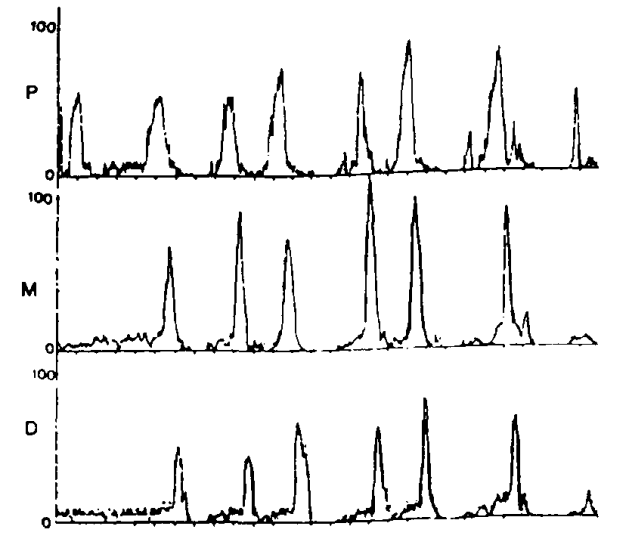

Fig. 2. The esophageal pressure waveform from the 5 samples $/ \mathrm{s}$ data recorded by the digital ambulatory monitor. Amplitude is in $\mathrm{mmHg}$; horizontal axis marks are 5 s. $P=$ proximal, $M=$ middle, $D=$ distal.

The analysis program detects esophageal contractile activity and calculates specific parameters such as peak amplitude, contraction duration, and propagation delay. Contraction duration is defined as the length of time and pressure wave is greater than $10 \mathrm{mmHg}$. Propagation delay is the elapsed time from threshold crossing on channel 1 to that on channel 3 . The results are tabulated, and the contractile events are categorized as normally propagated, simultaneous, retrograde, prolonged, or hypertensive contractions. Events that correspond to button presses are also characterized.

\section{Results AND Discussion}

For this pilot study of the monitoring system, we were interested in the incidence of occurrence of contractions that would be interpreted as abnormal in a standard motility laboratory. To define abnormalities, we consulted data from 85 normal subjects studied by Richter et al. [16] using standard stationary motility. This study of normals showed evidence that tertiary (simultaneous) contractions occurred in 18 percent of dry swallows. However, these are considered to be abnormal events in most clinical laboratories. We defined a simultaneous contraction to be one that has onset within $1 \mathrm{~s}$ in both the proximal and distal sensors. This is in keeping with the Richter et al. study of normals where the onset of the contrac- (a)

(b)

(c)

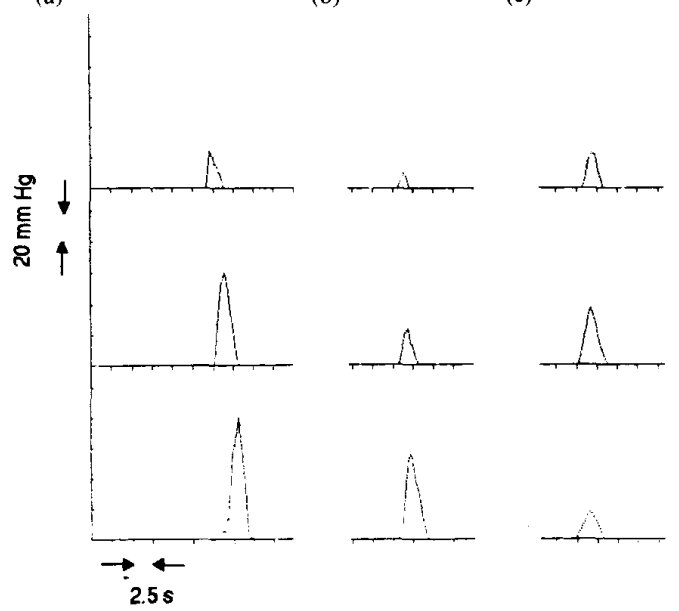

Fig. 3. Examples of (a) normal propagated contraction, (b) simultaneous contraction, and (c) retrograde contraction. Note that the contractions on each channel are measured from the uptake of the pressure wave.

tions in sensors spaced $10 \mathrm{~cm}$ apart should be within $1.8 \mathrm{~s}$. In our study, retrograde contractions were defined as contractions whose onset in the proximal lead followed the onset in the distal lead by greater $1 \mathrm{~s}$. Retrograde contractions are not seen in normals in stationary protocols. The amplitude of contractions is commonly used in standard clinical manometry to define abnormal patient populations. In most studies, an amplitude of $180 \mathrm{mmHg}$ is used as the upper limit cutoff. This is supported in the study of stationary normals. A duration of $10 \mathrm{~s}$ or greater was taken as our cutoff for a prolonged contraction. In the study of normals by Richter et al., the upper limit of normal would be somewhat less than $6 \mathrm{~s}$. Fig. 3 depicts examples of these "abnormal" events.

Ten normal subjects volunteered to undergo 24-h monitoring with the ambulatory device. After the subject's nasal cavity was numbed with 2 percent lidocain, the catheter was inserted transnasally into the esophagus and, while the pressure was monitored on a chart recorder, the catheter was positioned with transducers at 5,10 , and $15 \mathrm{~cm}$ above the lower esophageal sphincter. Recordings of ten wet swallows of $5 \mathrm{ml}$ water each were made on the chart recorder prior to connection of the catheter to the ambulatory mon- 
TABLE I

Frequency of Abnormal Contractile Events in the Ten Subjects

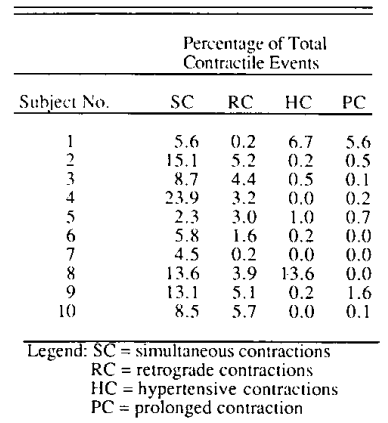

TABLE II

Averaged Characteristics of All Contractile Events for Proximal and Distal Channels for the Ten SubJects

\begin{tabular}{|c|c|c|c|c|c|}
\hline \multirow[b]{2}{*}{ Subj. no. } & \multicolumn{2}{|c|}{ Proximal channel } & \multicolumn{2}{|c|}{ Distal channel } & \multirow[b]{2}{*}{$\begin{array}{c}\text { Prop. delay } \\
\text { (s) }\end{array}$} \\
\hline & $\begin{array}{l}\text { Amplitude } \\
(\mathrm{mm} H \mathrm{Hg})\end{array}$ & \begin{tabular}{|c|}
$\begin{array}{c}\text { Duration } \\
(\mathrm{s})\end{array}$ \\
\end{tabular} & \begin{tabular}{|c|} 
Amplitude \\
(mmHg)
\end{tabular} & \begin{tabular}{|c}
$\begin{array}{c}\text { Duration } \\
(\mathrm{s})\end{array}$ \\
\end{tabular} & \\
\hline 1 & 39.2 & 1.8 & 69.2 & 2.8 & 1.7 \\
\hline 2 & 29.9 & 1.5 & 40.8 & 2.2 & 1.5 \\
\hline 3 & 23.1 & 1.2 & 46.9 & 2.0 & 1.7 \\
\hline 4 & 31.7 & 1.5 & 36.7 & 2.2 & 1.1 \\
\hline 5 & 46.4 & 1.5 & 57.2 & 2.6 & 2.5 \\
\hline 6 & 24.5 & 1.3 & 56.6 & 2.2 & 1.9 \\
\hline 7 & 27.8 & 1.5 & 39.2 & 1.7 & 2.6 \\
\hline 8 & 31.3 & 1.8 & 93.6 & 3.3 & 1.3 \\
\hline 9 & 31.4 & 1.6 & 44.3 & 2.9 & 2.1 \\
\hline 10 & 30.0 & 1.8 & 43.4 & 2.4 & 1.8 \\
\hline
\end{tabular}

itor. These were used to confirm that the contractile ability of the esophagus was normal.

The catheter was then connected to the portable monitor, which was strapped around the waist in a bicycle pack. Throughout the 24-h recording process, the subject was asked to push the button on the box once and then take a wet swallow of $5 \mathrm{ml}$ of water at regular intervals while awake. He/she was also asked to press the button twice each before and after a meal, three times before and after sleeping, and four times if anything unusual happened, such as food sticking in the esophagus or pain in the chest area, which was not expected to occur in normals. The subject was told to engage in any activities that the device did not interfere with and to eat anything that could be taken comfortably. After $24 \mathrm{~h}$, the subject returned, the catheter was removed, and the data were transferred to the IBM PC and analyzed.

Subject tolerance of the ambulatory device was generally good. However, two of the ten subjects asked that the device be removed before completion of the 24 -h monitoring period because of nasal discomfort and mild sore throat. Three others complained of a mild sore throat that resolved upon removal of the tube. We believe that much of this discomfort relates to the stiffness of the current catheter and will be diminished with the use of a more flexible catheter in the future.

Both manual and computer analysis of the data show that normal individuals often exhibit so-called "abnormal" contractile events at a higher rate than has been previously reported from conventional short-term manometric studies.

Table I shows the results of the categorization of the contractile events for the subjects. Many of the subjects showed fairly high incidences of one or more abnormal events. Table II gives the averaged characteristics from two of the three channels of the recognized legitimate peristaltic events. One subject showed an extremely high incidence of hypertersive contractions, indicative of the so-called "nutcracker" esophagus, but had no indication of pain. Table III is a comparison of the results of manual and computer analysis of the mean values of wet swallows of one subject. Computer analysis is clearly comparable to that of manual scoring.
TABLE III

Comparison of Manual Scoring and Computer Analysis in the Measlirement of the Characteristics of the Averages of 12 Wet SWALLOWS OF SUBJECT 5

\begin{tabular}{|c|c|c|}
\hline & $\begin{array}{l}\text { Manual } \\
\text { scoring }\end{array}$ & $\begin{array}{l}\text { Computer } \\
\text { analysis }\end{array}$ \\
\hline \multicolumn{3}{|c|}{ Anplitude $(\mathrm{mm} \mathrm{Hg})$} \\
\hline chan 1 & 49.8 & 48.4 \\
\hline $\begin{array}{l}\text { chan } 2 \\
\text { ches }\end{array}$ & 37.0 & $\begin{array}{l}4.4 \\
36.3\end{array}$ \\
\hline chan 3 & 93.2 & 93.2 \\
\hline \multicolumn{3}{|c|}{ Duration (s) } \\
\hline chan 1 & 1.6 & 1.5 \\
\hline chan 2 & 2.1 & 2.0 \\
\hline chan 3 & 2.7 & 2.7 \\
\hline \multicolumn{3}{|c|}{ Propagation delay $(s)$} \\
\hline & 2.5 & 2.4 \\
\hline
\end{tabular}

\section{Conclusions}

Overall, the system worked quite well with only minor technical difficulties. We found many contractile events in normals that would be considered abnormal in standard stationary motility. While the monitor and data processing programs should prove helpful in the evaluation of noncardiac chest pain subjects, the need for controls for comparison is clear. In the future, this type of ambulatory monitoring will likely become a standard method of diagnosis in the field of esophageal motility.

\section{REFERENCES}

[1] P. O. Katz, C. B. Dalton, J. E. Richter, W. C. Wu, and D. O. Castell, "Esophageal testing of patients with noncardiac chest pain or dysphagia," Ann. Intern. Med., vol. 106, pp. 593-597, 1987.

[2] S. B. Benjamin, D. C. Gerhardt, and D. O. Castell, "High amplitude, peristaltic esophageal contractions associated with chest pain and/or dysphagia," Gastroenterol., vol. 77, pp. 478-483, 1979.

[3] W. C. Orr and M. G. Robinson, "Hypertensive peristalsis in the pathogenesis of chest pain: Further exploration of the Nutcracker esophagus,'" Amer. J. Gastroenterol., vol. 77, pp. 604-607, 1982.

[4] M. Traube, R. Albibi, and R. W. McCallum, "High-amplitude peristaltic esophageal contractions associated with chest pain," J. Amer. Med. Ass., vol. 250, pp. 2655-2659, 1983.

[5] R. E. Clouse, A. Staiano, D. W. Landau, and J. L. Schlachter, "Manometric findings during spontaneous chest pain in patients with presumed esophageal 'spasm,', Gastroenterol., vol. 85, pp. 395$402,1983$.

[6] P. O. Katz and D. O. Castell, "Review: Esophageal motility disorders," Amer. J. Med. Sci., vol. 290, pp. 61-69, 1985.

[7] K. L. Koch, C. Curry, R. L. Feldman, C. J. Pepine, A. Long, and J. R. Mathias, "Ergonovine-induced esophageal spasm in patients with chest pain resembling angina pectoris," Dig. Dis. Sci., vol. 27, pp. 1073-1080, 1982.

[8] R. L. London, A. Ouyang, W. J. Snape, S. Goldberg, J. W. Hirschfeld, and S. Cohen, "Provocation of esophageal pain by ergonovine or edrophonium,"' Gastroenterol., vol. 81, pp. 10-14, 1981.

[9] J. J. Dalal, A. M. Dart, H. A. Daview, D. J. Sheridan, M. S. T. Ruttley, and A. H. Henderson, "Coronary and peripheral arterial responses to ergotamine in patients susceptible to coronary and oesophageal spasm," Br. Heart J., vol. 45, pp. 181-185, 1981.

[10] C. A. Lee, J. C. Reynolds, A. Ouyang, L. Baker, and S. Cohen, "Esophageal chest pain: Diagnostic value of high-dose tensilon provocative testing," Gastroenterol., vol. 86, p. 1156, 1984.

[11] R. M. Wexler and M. D. Kaye, "Pentagastrin in diffuse oesophageal spasm," Gut, vol. 22, pp. 212-216, 1981 .

[12] T. T. Nostrant, J. Sams, and T. Huber, "Bethanechol increases the diagnostic yield in patients with esophageal chest pain,"' Gastroenterol., vol. 91, pp. 1141-1146, 1986.

[13] J. Janssens, G. VanTrapen, and G. Ghillebert, "24-hour recording of esophageal pressure and $\mathrm{pH}$ in patients with noncardiac chest pain," Gastroenterol., vol. 90, pp. 1978-1984, 1986.

[14] L. J. Peters, L. C. Maas, C. B. Dalton, D. A. Petty, W. C. Wu, D. O. Castell, and J. E. Richter, "24-hour ambulatory combined esophageal motility $/ \mathrm{pH}$ monitoring in evaluation of non-cardiac chest pain (CP)," Gastroenterol., vol. 90, p. 1584, 1986. 
[15] R. B. Troxell, S. R. Kohn, J. E. Gray, R. W. Welch, E. D. Harloe, and R. K. Goyal, "A computer-assisted technique for 24-hour esophageal monitoring," Dig. Dis. Sci., vol. 27, pp. 1057-1062, 1982.

[16] J. E. Richter, W. C. Wu, D. N. Johns, J. N. Blackwell, J. L. Nelson, J. A. Castell, and D. O. Castell, "Esophageal manometry in 95 healthy adult volunteers: Variability of pressures with age and frequency of 'abnormal' contractions," Dig. Dis. Sci. . vol. 32, pp. 583$592,1987$.

\section{An Improved Buffer for Bioelectric Signals}

RAMON PALLAS-ARENY, JOSEP COLOMINAS, AND JAVIER ROSELL

\begin{abstract}
We propose an ac-coupled amplifier that offers a high input impedance, thus making it suitable for bioelectric signal amplification. We also present the necessary formulas for calculating its input impedance and transfer function in order to facilitate its adaptation to different applications.
\end{abstract}

\section{INTRODUCTION}

The amplification of bioelectric signals requires them to be ac coupled before high gain amplification, either to avoid saturations due to contact potentials arising at electrode-electrolyte interfaces [1] or for further band-limiting purposes. At the same time, in order to reduce interferences due to the usually high electrode-skin impedance and its variability [2], the amplifier needs to have a very high input impedance. This calls for an input stage acting as buffer.

In most designs, each one of these functions-ac coupling and buffering-is implemented at different stages of the circuit. In this work, we prove, both analytically and experimentally, that it is possible to merge both functions in a single stage. We also present the formulas enabling us to apply the proposed circuit to different signals in order to tailor it to particular needs.

\section{AC Coupling and InPut Impedance in Bioelectric} SigNAL AMPLIFIERS

\section{A. AC Coupling}

In order to measure bioelectric signals, it is necessary to use electrodes. At any electrode-electrolyte interface, a contact potential arises, whose magnitude is very high and subject to variations due to many parameters [1]. Thus, in spite of two electrodes being involved in any biopotential measurement, the prospect of a tight balance between them is so remote that, when designing the amplifier, the possible presence of a dc potential higher in magnitude than the expected signal must be taken into consideration. The measurement is certainly a differential one, but contact potentials would seldom cancel each other out.

The usual solution is to decouple dc voltages to prevent offset potentials from saturating the amplifier whenever its voltage gain has a value of $40 \mathrm{~dB}$ or even lower. This need of an ac coupling is a serious drawback for various reasons. In low-noise design, for example, it is important to have as much gain as possible concentrated in the first stage of the amplifier. Bioelectric signals normally require voltage gains in excess of $60 \mathrm{~dB}$.

Therefore, if ac coupling cannot be implemented before the first amplifier, as happens with ordinary circuits, the designer is prevented from taking advantage of the improved performances of

Manuscript received March 8, 1988; revised September 26, 1988.

The authors are with the Division de Instrumentacion y Bioingenieria, Departamento de Ingeniería Electrónica, U.P.C., ETS Ing Telecom, P.O. Box 30002,08080 Barcelona, Spain.

IEEE Log Namber 8825623.

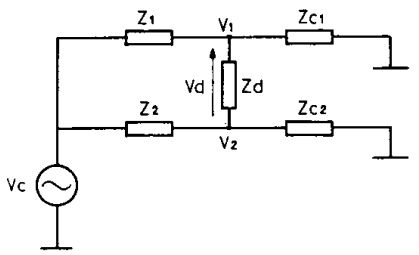

Fig. 1. Circuit model for the input of a biopotential amplifier showing how any imbalance in the front end converts a common mode signal into a differential one. The imbalance can be on the electrode side or on the amplifier side.

modern operational amplifiers which offer very low noise levels because he or she is compelled to postpone high-voltage gains until an ac signal is obtained [3]. Furthermore, for a given tolerance in components, the CMRR attained increases if voltage gain is large in differential stages, although it is unaffected by voltage gain in nondifferential ones.

Also, when dealing with several electrodes and only one or a few differential amplifiers, it is necessary to place a buffer for each electrode. If they are dc coupled, it may happen that a saturation occurs in the amplifier when switching from one lead to another because of electrode offset potentials. Therefore, some provision must be made in order to rapidly restore voltage levels. An accoupled buffer might eliminate this problem.

\section{B. Input Impedances}

Another requirement when measuring bioelectric signals is the need for differential amplifiers to have very high input impedances, both differential and common mode. A high differential mode input impedance minimizes loading effects. A high common mode input impedance reduces the differential mode voltage that results from common mode input signals [2]. This common mode-to-differential mode conversion results from any imbalance between both input terminals, as indicated in Fig. 1, and shows up as an increased interference at power line frequency.

In Fig. $1, Z_{1}$ and $Z_{2}$ are any impedances in front of the amplifier. Whenever either $Z_{1} \neq Z_{2}$ or $Z_{c 1} \neq Z_{c 2}$, the former being the usual situation, then $v_{1} \neq v_{2}$, and an interfering signal will result. In most designs, $Z_{1}$ and $Z_{2}$ are mainly attributable to electrode-skin impedances. The resulting interference can be effectively reduced by making $Z_{c 1}$ and $Z_{c 2}$ very high. Buffering each electrode with a unity gain voltage follower or a buffer with gain is the usual solution.

The placement of a capacitor in series with each electrode would certainly block any dc voltage, and therefore might be considered for decoupling. However, there is a simultaneous need for a path for $\mathrm{dc}$ bias currents entering the amplifier, and the blocking capacitor would prevent any dc current from coming through the electrodes. Placing a resistor from each input of the amplifier to ground would solve this problem, but it would be necessary to use rather unusual components in order to obtain the high input impedance required for a bearable interference and the optimum low-frequency response.

For these reasons, typical biopotential amplifiers have a dc-coupled moderate-gain input stage, thus biasing through the electrodes and reducing the risk of saturation at this stage. Afterwards-preferably once dealing with a nondifferential signal-an ac-coupled stage offering an additional gain is put in place. A typical circuit is the one proposed by Neuman [4].

\section{ANalysis of an AC-Coupled Buffer}

In accordance with the above reasons, the placement of a series capacitor in front of an amplifier will be a satisfactory solution, provided that a suitable way of biasing the circuit is offered. This 